\title{
Semeadura Direta para Restaurar Savanas Neotropicais: uma Alternativa para Adequação Ambiental no Cerrado
}

\author{
Ana Wiederhecker Gabriel ${ }^{1}$, Eric Scaramello do Nascimento Silva ${ }^{1}$, Miguel de Freitas Sartori ${ }^{2}$, Isabel Belloni Schmidt ${ }^{3} \&$
} Alexandre Bonesso Sampaio ${ }^{1}$

\author{
Recebido em 25/11/2021 - Aceito em 07/04/2021 \\ ${ }^{1}$ Instituto Chico Mendes de Conservação da Biodiversidade/ICMBio, Brasil. <ana.wiederhecker@gmail.com, eric.scaramello@gmail.com, \\ alexandre.sampaio@icmbio.gov.br > \\ 2 Agência Reguladora de Águas/ADASA, Brasil. < miguel.sartori@adasa.df.gov.br> \\ ${ }^{3}$ Universidade de Brasilia/UNB, Brasil. <isabelbschmidt@gmail.com>
}

\begin{abstract}
RESUMO - Restaurações de savanas neotropicais são muitas vezes realizadas de forma inadequada, desconsiderando características ecológicas dessas vegetações. Ameaçadas pela conversão para atividades agrícolas e introdução de espécies invasoras, a conservação desses ambientes depende da restauração ecológica. Em locais com presença de espécies exóticas invasoras, o monitoramento e o manejo após a restauração são de extrema importância, sendo frequentemente negligenciados. O presente estudo foi realizado em sete áreas onde houve restauração por semeadura direta de espécies arbóreas, arbustivas e herbáceas nativas de formações savânicas do Cerrado. Em cada uma das áreas, indicadores de: cobertura vegetal, densidade e riqueza de espécies nativas regenerantes foram amostrados pela disposição de parcelas de $100 \mathrm{~m}^{2}$. Os resultados obtidos foram avaliados de acordo com os valores de referência estabelecidos pela norma de restauração do DF. O histórico das áreas e o preparo do solo pareceram interferir de maneira significativa nos caminhos da restauração, afetando a composição da comunidade vegetal e os valores obtidos para os indicadores amostrados em cada área. Os valores de referência definidos pela norma do DF não foram alcançados integralmente por nenhuma das áreas. Ainda assim, os resultados indicam que é possível atingir os requerimentos legais para cobertura de plantas nativas, densidade e riqueza de regenerantes por meio da restauração ecológica. Para isso, o revolvimento do solo pode ser utilizado para o controle das invasoras e a semeadura direta de gramíneas, arbustos e árvores para a reitrodução da biodiversidade nativa. Para o sucesso ser alcançado, é necessária a melhoria no controle das espécies invasoras antes da semeadura, bem como omonitoramento e a manutenção.
\end{abstract}

Palavras-chave: Recuperação de áreas degradadas; recomposição da vegetação nativa; ecossistemas graminosos; invasão biológica.

\section{Direct Seeding as a Way for Neotropical Savannas Restoration: an Alternative for Cerrado's Environmental Compliance}

\begin{abstract}
In neotropical savannas, ecosystems are often restored inefficiently due to practitioners overlooking innate ecological characteristics of their vegetation. Threatened by land conversion and biological invasions, these ecosystems are in need of efficient restoration efforts. In places where exotic species are found, post restoration monitoring and management is highly important, though often overlooked. The present study took place in seven areas under restoration by direct seeding of native trees, shrubs and grasses of the Cerrado biome. Each area was surveyed by analysis of vegetation cover, density and richness of native regenerant species per area by sampling $100 \mathrm{~m}^{2}$ plots. The data acquired was then analyzed considering the indicator thresholds for restorations success established by Federal District legislation. Past area history as well as soil preparation were found to significantly interfere in the restoration pathways, directly affecting plant community composition and resulting in differences between the study areas. No area fully achieved the legislation requirements. However, our results indicate that these requirements can be achieved by ecological restoration. For that purpose, soil tillage can be used to control weeds and direct sowing of grasses, shrubs and trees to reintroduce native biodiversity. For success to be achieved, it is necessary to improve the control of invasive species before sowing, as
\end{abstract}


well as monitoring and maintenance.

Keywords: Recuperation of degraded areas; native vegetation recomposition; grassy ecosystems, biological invasions.

\section{Siembra Directa para Restaurar Sabanas Neotropicales: una Alternativa para la Adecuación Ambiental en el Cerrado}

RESUMEN - Las restauraciones de las sabanas neotropicales a menudo se realizan de manera inadecuada, sin tener en cuenta las características ecológicas de estas vegetaciones. Amenazados por la conversión a actividades agrícolas y la introducción de especies invasoras, la conservación de estos ambientes depende de la restauración. En lugares con presencia de especies exóticas invasoras, el monitoreo y manejo de las invasoras después de plantar es extremadamente importante. El presente estudio se llevó a cabo en siete áreas donde hubo restauración por siembra directa de especies arbóreas, arbustivas y herbáceas nativas de formaciones de sabana en el Cerrado. En cada una de las áreas se muestrearon indicadores de: cobertura vegetal, densidad y riqueza de especies nativas en regeneración. Los resultados obtenidos se evaluaron de acuerdo con los valores de referencia establecidos por el estándar de restauración del DF. La historia de las áreas y la preparación del suelo parecieron interferir significativamente en los caminos de restauración, afectando la composición de la comunidad vegetal. Los valores de referencia definidos por la norma del DF no fueron alcanzados en su totalidad por ninguna de las áreas. Aun así, los resultados indican que es posible cumplir con los requisitos legales de cobertura vegetal nativa, densidad y riqueza de regenerantes a través de la restauración ecológica. Para ello, la preparación del suelo se puede utilizar para controlar las gramineas invasoras y la siembra directa de gramineas, arbustos y árboles para reintroducir la biodiversidad nativa. Para lograr el éxito es necesario mejorar el control de las especies invasoras antes de la siembra, así como el monitoreo y mantenimiento.

Palabras clave: Recuperación de áreas degradadas; recomposición de la vegetación nativa; ecosistemas herbáceos; invasión biológica.

\section{Introdução}

Por todo o globo, severas mudanças ambientais induzidas por seres humanos têm originado ecossistemas alternativos, que produzem menos serviços ambientais, são menos diversos e dominados por espécies exóticas, em que a regeneração natural de plantas nativas dificilmente ocorre sem a intervenção humana (Buisson et al., 2019; Suding, 2011). Nesse cenário mundial, a restauração ecológica tem sido vista como um elemento chave para o alcance de metas globais de conservação, além de uma importante ferramenta de mitigação e reversão das mudanças impostas aos ambientes naturais (Hobbs et al., 2011; Harris \& Diggelen, 2006). Consequentemente, tem sido gerada uma alta demanda por projetos de restauração levando a um rápido desenvolvimento dessa área da ciência (Wortley et al., 2013; Suding, 2011). Não obstante, a baixa quantidade de estudos que avaliam o sucesso $e$ as falhas de projetos de restauração, ou monitoram seus resultados, faz da restauração ecológica uma área com muitas lacunas de conhecimento (Schmidt et al., 2019; Suding, 2011), especialmente em savanas (Wortley et al., 2013).

Uma importante lacuna a ser superada é o controle de espécies exóticas invasoras (EEI). Consideradas uma ameaça global à biodiversidade, espécies exóticas são atualmente reconhecidas como um dos maiores obstáculos para a restauração, pois possuem características que dificultam sua erradicação em áreas invadidas (Sampaio \& Schmidt, 2014; D’Antonio \& Vitousek, 1992). Sua grande distribuição torna as EEI um dos maiores desafios, em extensão, para a restauração ecológica (Cava et al., 2016; Sampaio \& Schmidt, 2014; Hoffmann \& Haridasan, 2008). Ademais, espécies invasoras, são capazes de alterar processos do solo, microclima regional e distúrbios endógenos das áreas que ocupam impedindo o restabelecimento da vegetação nativa (Buisson et al., 2019; Damasceno et al., 2018; Sampaio \& Schmidt, 2014; D’Antônio \& Vitousek, 1992). 
O controle mecânico tem sido comumente aplicado para o manejo de gramíneas exóticas invasoras (GEI) visando restauração dos ecossistemas naturais. Em áreas de pastagem onde tais espécies foram semeadas após remoção da vegetação nativa, preparo do solo e adubação, o revolvimento mecânico do solo pode auxiliar em seu controle. Esse procedimento, ao mesmo tempo que arranca as plantas invasoras pela raiz, promove a germinação do banco de sementes. Além disso, há a possibilidade dessa técnica diminuir os nutrientes disponíveis, o que pode favorecer as plantas nativas adaptadas a situações de baixa fertilidade. Aplicada sucessivamente, com intervalos de tempo, essa técnica também permite o esgotamento do banco de sementes das invasoras ao promover a germinação em uma primeira aplicação e o arranquio das plântulas na próxima (Coutinho et al., 2019).

Em áreas de vegetação campestre e savânica, como no Cerrado, uma vez reduzida a presença de gramíneas invasoras, é necessário reintroduzir as plantas nativas. Elas apresentam baixa taxa de dispersão, mesmo na presença de áreas de vegetação nativa próximas, o que torna a regeneração natural improvável (Silveira et al., 2020). Para a restauração desses tipos de vegetação, é necessário restabelecer o estrato herbáceo-arbustivo, para isso tem sido utilizada a técnica da semeadura direta, que permite o plantio em escala de gramíneas e arbustos nativos (Sampaio et al., 2015).

Protocolos para armazenagem e coleta das sementes são escassos e pouco abordados em estudos (Schmidt et al., 2018). Não obstante, a técnica de semeadura direta permite a introdução simultânea de um razoável número de espécies com diferentes formas de crescimento (Sampaio et al., 2019; Pellizzaro et al., 2017; Palma \& Laurance, 2015). Contribui-se, assim, para o aumento da diversidade da área restaurada. Espécies de gramíneas e arbustos ocupam rapidamente os nichos disponíveis controlando as exóticas e permitindo o desenvolvimento das espécies arbóreas de crescimento lento (De Oliveira et al., 2019; Pellizzaro et al., 2017; Pilon \& Durigan, 2013). Entretanto, a redução da cobertura de exóticas não indica, necessariamente, sucesso para a restauração (Assis et al., 2020). Nos primeiros anos, a cobertura vegetal de uma área em restauração pode evoluir tanto para a predominância de nativas como para a de exóticas (Coutinho et al., 2019), o que pode depender da pressão de propágulos das invasoras.
A restauração de fitofisionomias savânicas e campestres é ainda pouco conhecida. Para que seja possível o desenvolvimento de técnicas e dos fatores que possam levar ao sucesso, ações de monitoramento e manutenção são imprescindíveis (Buisson et al., 2019). Até o presente momento, o sucesso ou falha de uma restauração é avaliado por parâmetros de diversidade e estrutura da vegetação, assim como por processos ecossistêmicos. Esses parâmetros sinalizam o retorno da regeneração natural ou permanência do estado de degradação (Sampaio et al., 2015, Ruiz-Jaen \& Aide, 2005). A interferência humana em um ecossistema pode ter resultados irregulares (Hobbs \& Cramer, 2008), principalmente em áreas onde a presença de espécies exóticas resultou em estados alternativos de ecossistemas. Nessas áreas, o restabelecimento da diversidade de espécies vegetais é lento (Buisson et al., 2019). Reinvasões são recorrentes (Sampaio et al, 2015) e o manejo contínuo para o controle das GEI é, muitas vezes, necessário (Kettenring \& Adams, 2011).

O presente estudo teve por objetivo avaliar o sucesso da restauração por semeadura direta em área originalmente coberta por vegetação de cerrado na Reserva Biológica do Rio Descoberto, DF. Buscouse também propor intervenções para aumentar o sucesso da restauração.

\section{Materiais e Métodos}

O presente estudo foi conduzido na Reserva Biológica do Descoberto (15²7'42" Sul; 48 21'82" Oeste, datum:WGS84), DF, Brasil. Nessa região há predominância de Latossolo VermelhoAmarelo e o clima é Tropical Aw pela classificação de Köppen (Reatto et al., 2004).

O estudo consistiu na avaliação do sucesso da restauração de 16,8ha (Fig. 1), às margens da barragem do rio Descoberto, divididos em sete áreas seguindo o Protocolo de Monitoramento da Vegetação Nativa do Distrito Federal (Souza \& Vieira, 2017). A amostragem de acordo com este Protocolo foi estratificada e, para isso, foram definidas as sete áreas caracterizadas por: localização, histórico de uso e técnica de restauração aplicada. Em todas as áreas, a vegetação original, cerrado sentido restrito, havia sido convertida em pastagens para criação de animais. Entre os anos de 2018 e 2019, as áreas receberam ações de restauração por semeadura direta e plantio de mudas para controle de exóticas e recuperação da cobertura de nativas. 


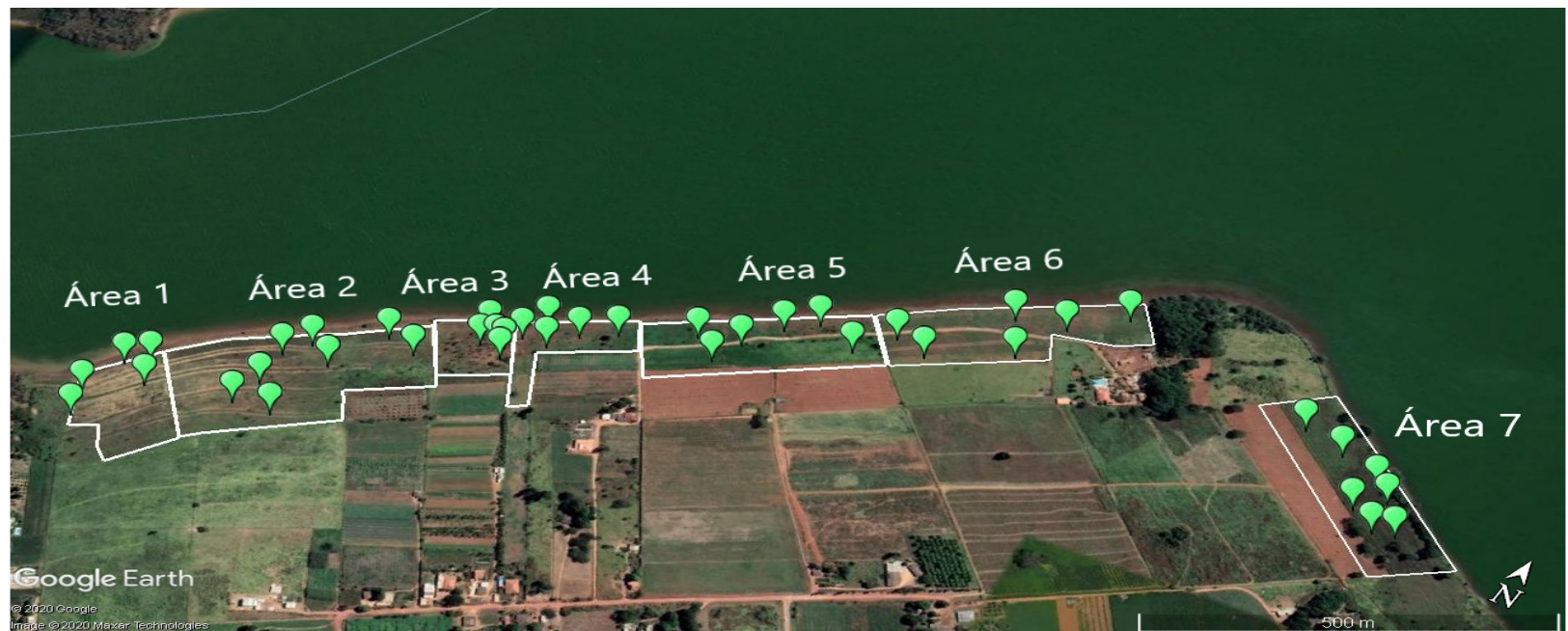

Figura 1 - Mapa das áreas amostradas na Reserva Biológica do Descoberto entre julho de 2019 e janeiro de 2020. As linhas brancas indicam o perímetro de cada área e os localizadores o ponto inicial de cada parcela.

As ações de semeadura foram realizadas em dois momentos distintos. Primeiramente, em janeiro de 2018, a Área 1 recebeu o plantio de 1500 mudas em linhas concentradas em uma parte da área e semeadura direta manual nas entrelinhas (Tabela 1). Entre dezembro do mesmo ano e janeiro de 2019, essa área recebeu semeadura de adensamento manual e roçagem do capim exótico. Nesse mesmo período, as áreas
2 a 7, após preparo do solo, receberam semeadura direta mecanizada, pela utilização de distribuidoras de calcário distribuindo nas áreas uma mistura de terra e sementes. Em todas as áreas, foram introduzidos, por semeadura direta mecanizada: arbustos, subarbustos, ervas e árvores. Desde então, essas áreas receberam acompanhamento voltado para a quantificação visual e erradicação das plantas invasoras por capinas manuais.

Tabela 1 - Lista das áreas amostradas na Reserva Biológica do Descoberto. O histórico é referente aos últimos 10 anos antes da intervenção.

\begin{tabular}{|c|c|c|c|c|c|c|c|c|}
\hline \multirow[t]{2}{*}{ Área } & \multirow[t]{2}{*}{ Hectares } & \multirow[t]{2}{*}{$\begin{array}{l}\text { Número de } \\
\text { parcelas }\end{array}$} & \multirow[t]{2}{*}{ Histórico } & \multirow[t]{2}{*}{ Plantio } & \multicolumn{3}{|c|}{ Preparo } & \multirow[t]{2}{*}{$\begin{array}{l}\text { Sementes } \\
\qquad / \mathbf{m}^{2}\end{array}$} \\
\hline & & & & & $\begin{array}{l}\text { Número de } \\
\text { gradagens }\end{array}$ & $\begin{array}{l}\text { Número de } \\
\text { nivelamentos }\end{array}$ & Outros manejos & \\
\hline 1 & 1,7 & 5 & Pastagem de Braquiária & 2018 & 1 & 2 & $\begin{array}{c}\text { Queimada natural, } \\
\text { bacia de contenção, } \\
\text { terraceamento e curva } \\
\text { de nível. }\end{array}$ & $441^{*}$ \\
\hline 2 & 4,5 & 8 & Pastagem de Braquiária & 2019 & 6 & 0 & $\begin{array}{c}\text { Capina seletiva e curva } \\
\text { de nível. }\end{array}$ & 765 \\
\hline 3 & 0,9 & 5 & $\begin{array}{l}\text { Pastagem de Andropogon } \\
\text { sp. com Cerrado } \\
\text { remanescente e elevada } \\
\text { densidade de árvores }\end{array}$ & 2019 & 6 & 0 & Capina seletiva. & 765 \\
\hline 4 & 1,1 & 5 & $\begin{array}{l}\text { Pastagem de Braquiária } \\
\text { com Cerrado } \\
\text { remanescente }\end{array}$ & 2019 & 5 & 1 & $\begin{array}{l}\text { Queima e capina } \\
\text { seletiva. }\end{array}$ & 765 \\
\hline 5 & 2,7 & 6 & $\begin{array}{l}\text { Pastagem de Braquiária } \\
\text { com bovinos e adubação }\end{array}$ & 2019 & 3 & 1 & & 765 \\
\hline 6 & 2,8 & 6 & $\begin{array}{l}\text { Pastagem de Braquiária } \\
\text { com caprinos }\end{array}$ & 2019 & 5 & 1 & & 765 \\
\hline 7 & 3,1 & 7 & Pastagem de Braquiária & 2019 & 5 & 1 & Capina seletiva & 765 \\
\hline
\end{tabular}

*Na área 1 foi realizada uma semeadura de adensamento com 928 sementes $/ \mathrm{m}^{2}$ e foram plantadas 1500 mudas. 


\section{Desenho experimental}

As áreas foram amostradas entre junho de 2019 e janeiro de 2020. A distribuição das parcelas por área foi realizada de forma aleatória utilizando a função de seleção aleatória de pontos em uma grade do Quantum GIS (QGIS) (Sherman et al., 2011), e o número de parcelas foi estabelecido segundo o Protocolo de Monitoramento da Recomposição da Vegetação Nativa do Distrito Federal (Sousa \& Vieira, 2017). Cada parcela correspondia a um retângulo de $25 \mathrm{~m} \mathrm{X} 4 \mathrm{~m}$ de largura, totalizando $100 \mathrm{~m}^{2}$.

Foram três os parâmetros amostrados em cada parcela: cobertura vegetal, densidade $e$ diversidade de espécies regenerantes de plantas lenhosas nativas. A cobertura vegetal foi amostrada pela utilização de trena de $25 \mathrm{~m}$ e uma vara de bambu para aplicação do método de interceptação de ponto em linha (Sousa \& Vieira, 2017), totalizando 26 pontos de amostragem por parcela, um a cada metro. A densidade de espécies regenerantes nativas foi amostrada pela contagem de indivíduos regenerantes (entre $30 \mathrm{~cm}$ e $2 \mathrm{~m}$ de altura) por espécie e parcela. Por fim, a diversidade de nativas foi amostrada pela contagem do número de espécies por unidade de área. Para identificação, foram coletados dois exemplares botânicos de cada espécie não conhecida, as quais foram secas em estufa para produção de exsicatas.

\section{Análise de dados}

Para o cálculo de cobertura, densidade de regenerantes e número de espécies foi seguido o Protocolo de Monitoramento da Recomposição da Vegetação Nativa do Distrito Federal (Sousa \& Vieira, 2017). A caracterização da cobertura vegetal de cada área foi realizada pela definição de cinco categorias: gramínea exótica (GE), gramínea nativa (GN), lenhosa nativa (LN), nativa total (NT) e sem vegetação $(\mathrm{SV})$. Os toques de cada uma dessas categorias foram contabilizados nos 26 pontos ao longo da transecção de $25 \mathrm{~m}$ e convertidos em porcentagem de cobertura. Os resultados das análises de cobertura foram adicionalmente submetidos a análises estatísticas para testar diferenças significativas entre as áreas. Tal análise foi realizada utilizando o método Generalized Linear Models (GLM), com distribuição de
Poisson, para cada categoria de cobertura do solo. Ademais, foram realizados testes pareados de hipótese linear geral (GLHT) para as categorias que apresentaram diferenças significativas ( $p<$ $0,05)$ entre as áreas. As distribuições de Poisson e GLHT foram calculadas utilizando os pacotes do programa $\mathrm{R}$ base e multcomp, respectivamente ( $\mathrm{R}$ Core Team, 2018; Hothorn, 2008).

Após as análises, os valores de diversidade de espécies, cobertura por espécies exóticas $e$ nativas, assim como a densidade de regenerantes, foram comparados com os valores de referência definidos na Instrução Normativa do Instituto Brasília Ambiental (IBRAM) $\mathrm{n}^{\circ}$ 723, de 22 de novembro de 2017 (IBRAM, 2017) e na Nota Técnica IBRAM no. 1/2018 (Tabela 2). Para calcular diferenças significativas entre as coberturas médias por categoria e o índice estabelecido pela legislação, foram realizados testes $\mathrm{T}$ com correção de Bonferroni, usando a função t.test do programa R (R Core Team, 2018; Hothorn, 2008).

Tabela 2 - Indicadores ecológicos para vegetação Savânica em Unidades de Conservação de Proteção Integral (IBRAM, 2017).

\begin{tabular}{|l|l|}
\hline Indicadores ecológicos & Valor \\
\hline Cobertura total (porcentagem mínima) & 80 \\
\hline $\begin{array}{l}\text { Cobertura de espécies exóticas perenes ou ciclo } \\
\text { longo (porcentagem máxima) }\end{array}$ & 0 \\
\hline $\begin{array}{l}\text { Cobertura gramíneas exóticas } \\
\text { (porcentagem máxima) }\end{array}$ & 40 \\
\hline $\begin{array}{l}\text { Cobertura vegetação lenhosa nativa } \\
\text { (porcentagem mínima) }\end{array}$ & 30 \\
\hline $\begin{array}{l}\text { Cobertura gramíneas nativas } \\
\text { (porcentagem mínima) }\end{array}$ & 30 \\
\hline $\begin{array}{l}\text { Cobertura vegetação nativa } \\
\text { (porcentagem mínima) }\end{array}$ & 80 \\
\hline $\begin{array}{l}\text { Densidade de regenerantes nativos } \\
\text { (indivíduos/hectare) }\end{array}$ & 3000 \\
\hline $\begin{array}{l}\text { Número de espécies nativas } \\
\text { (valor mínimo, áreas } \leq 7 h a)\end{array}$ & 20 \\
\hline
\end{tabular}

Fonte: Instituto Brasília Ambiental (IBRAM), Indicadores Ecológicos Para a Recomposição da Vegetação Nativa no Distrito Federal, 2017. 
Para avaliar a diversidade de espécies entre as áreas, foram calculados os índices de diversidade de Simpson (representado como 1-D) e de similaridade de SФrensen (Ss), curvas de acúmulo e número de espécies dominantes. Os índices de diversidade e similaridade $e$ as curvas de rarefação foram calculados utilizando o pacote vegan do programa $\mathrm{R}$ (Oksanen et al., 2019) $e$ as espécies dominantes em cada área foram definidas como aquelas com frequência superior a $1 / S$ sendo $S$ o número total de espécies na comunidade (Uramoto et al., 2005).

A identificação das espécies coletadas foi feita por especialistas e comparação com exsicatas de herbário no Laboratório de Ecologia Vegetal da Universidade de Brasília. Os nomes das espécies foram escritos com base na nomenclatura adotada pela Flora do Brasil 2020 (Flora do Brasil 2020, em construção).

\section{Resultados}

Nenhuma das áreas atingiu os requerimentos normativos integralmente. Seis das sete áreas (2, 3, 4, 5, 6 e 7), contudo, muito se aproximaram desse objetivo, alcançando entre 55 e $75 \%$ dos valores de cobertura exigidos. Nas áreas 1 a 4, a cobertura de exóticas amostrada, originalmente maior que $80 \%$, foi inferior ou próxima ao limite de $40 \%$ permitido pela legislação, não havendo diferenças significativas entre essas áreas (Fig. 2). Nas áreas 5, 6 e 7, por outro lado, a cobertura de exóticas foi, em média, $23 \%$ superior ao permitido, diferenciando significativamente das demais áreas que não a área 1 . Apesar disso, mais do que $50 \%$ dos requisitos legislativos foram cumpridos nessas áreas, tendo somente a área 7 apresentado uma cobertura significativamente maior do que o indicado pela legislação.
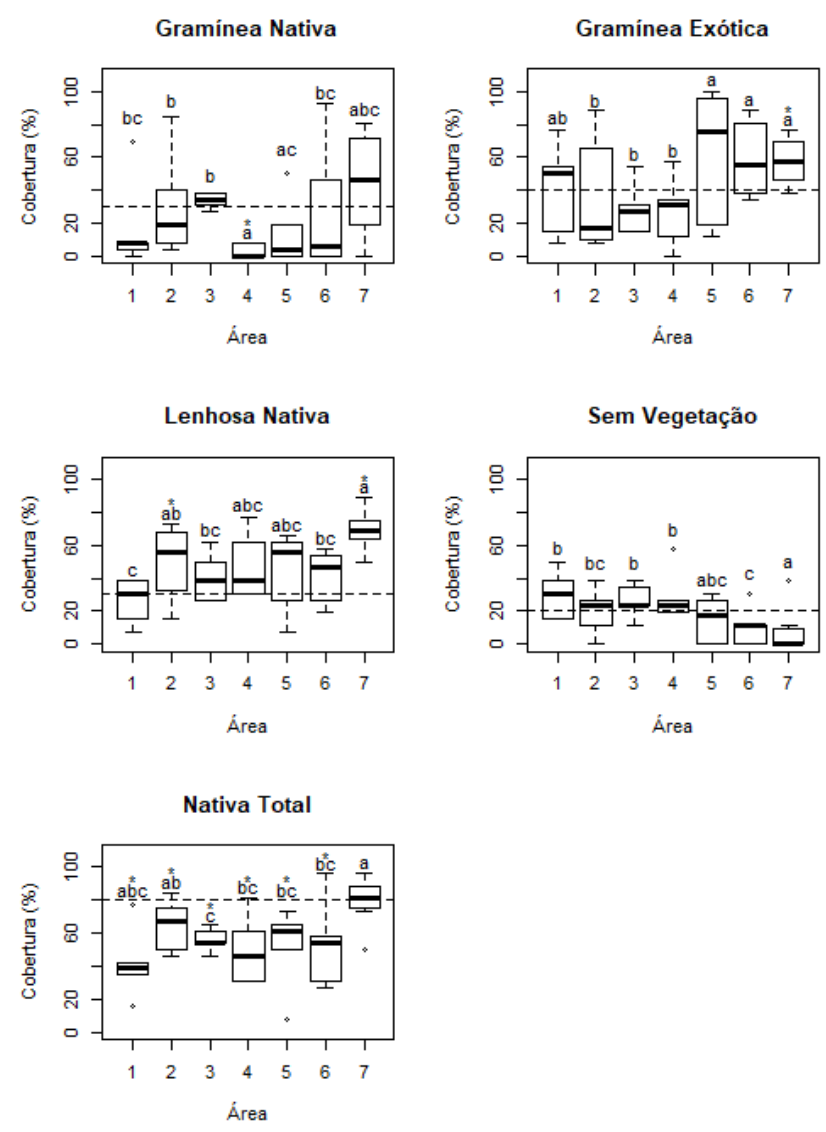

Figura 2 - Porcentagem de cobertura vegetal amostrada por área na Reserva Biológica do Descoberto coletadas por categoria: gramínea exótica, gramínea nativa, lenhosa nativa, nativa total e sem vegetação. As linhas tracejadas horizontais representam os parâmetros de cobertura estabelecidos pelo IBRAM. Diferentes letras indicam diferenças significativas entre os valores $(p<0,05)$. Asteriscos representam diferenças significativas em relação a linha tracejada $(p<0,05)$. Os retângulos indicam os $1^{\circ}, 2^{\circ} e$ $3^{\circ}$ quartis e as linhas tracejadas verticais indicam os valores mínimos e máximos, os pontos indicam valores extremos (outliers). 
Para todas as áreas, a porcentagem de solo descoberto (SV) foi inferior ou igual a $30 \%$. Somente três áreas se encontram fora do indicado pela legislação para essa categoria. Ainda assim, não foram encontradas diferenças significativas para essa categoria de cobertura. As gramíneas nativas (GN) cobriram menos de $30 \%$ do solo em $70 \%$ das áreas, apresentando as maiores variações nas porcentagens de cobertura. Os percentuais de cobertura por GN na área 4 foram significativamente menores do que os $30 \%$ exigidos pela legislação. A cobertura por lenhosas nativas (LN) foi superior em pelo menos $10 \%$ ao exigido, em todas as áreas com exceção da área 1. Nessa área, a cobertura foi $4 \%$ inferior ao exigido sem, contudo, apresentar diferenças significativas do valor exigido. Nas áreas 2 e 7, por outro lado, a cobertura de LN foi significativamente maior quando comparada às áreas 1, 3 e 6 e à legislação. Ainda assim, a cobertura de nativas total (NT), em média $58 \%$, apresentou-se abaixo do requerido pela legislação em $86 \%$ das áreas. Com exceção da área 7, todas as áreas apresentaram cobertura por NT significativamente menor do que o exigido na norma.

Em todas as áreas foram encontradas 249 espécies, 44 foram identificadas em nível de espécie e 38 em nível de família (ver material suplementar). Dessas, somente 8 foram semeadas, sendo 74 provenientes de mecanismos de regeneração natural. Nenhuma das mudas plantadas na Área 1 foram amostradas. Ademais, para as áreas 1, 5 e 7, as análises de frequência revelaram que inúmeras espécies nativas abundantes nessas áreas (Fig. 3) não são oriundas de semeadura tendo colonizado a área naturalmente após redução das exóticas. $\mathrm{Na}$ área 7, especificamente, houve grande abundância de uma espécie não identificada com indivíduos maiores e menores que $2 \mathrm{~m}$. Nas demais áreas, por outro lado, a dominância apresentou-se restrita a três espécies semeadas.

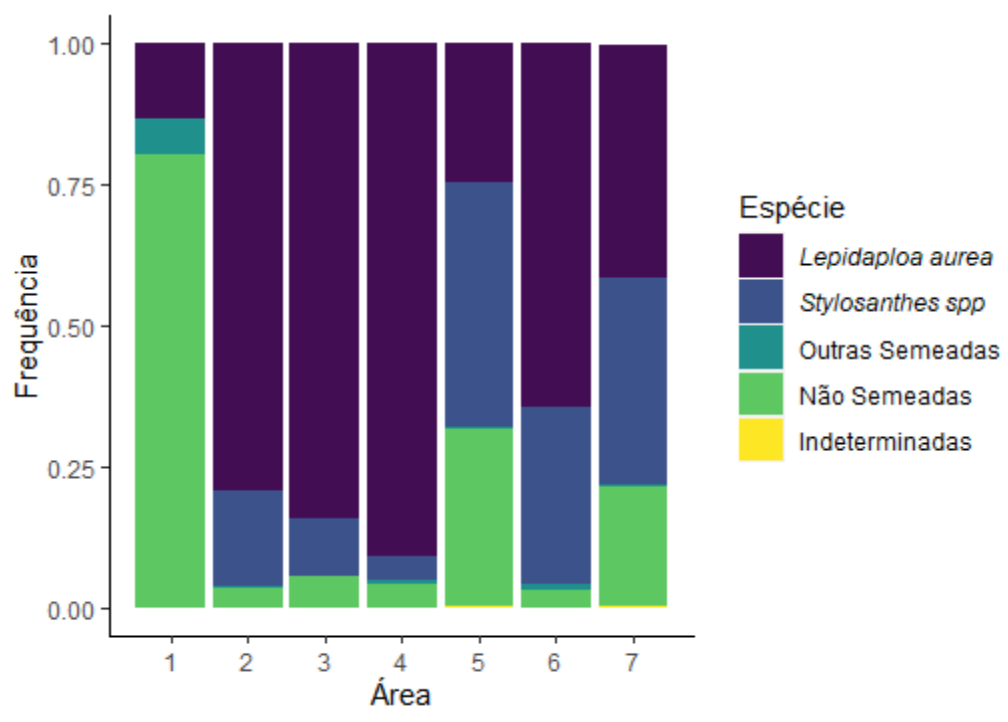

Figura 3 - Frequência das espécies lenhosas nativas encontradas em cada uma das sete áreas amostradas na Reserva Biológica do Descoberto entre junho de 2019 e janeiro de 2020. As barras representam a frequência de presença de cada grupo. O grupo indicado por Stylosanthes spp. é composto pelas duas espécies de Stylosanthes semeadas, sendo elas: Stylosanthes capitata Vogel e Stylosanthes macrocephala M.B. Ferreira \& Sousa Costa.

Para o indicador de riqueza de espécies regenerantes, todas as áreas atingiram valores acima do mínimo de 20 espécies. As áreas 1, 5 e 7 apresentaram em média 60 espécies e as demais em média 33 espécies (Tabela 3). O índice de diversidade de Simpson indica um padrão diferente da riqueza devido à diferente equitabilidade de espécies entre as áreas. A área 1 apresentou maior equitabilidade que as demais $e$, por isso, maior diversidade, apesar de não apresentar a maior riqueza. As curvas de rarefação indicam o mesmo padrão encontrado para o número de espécies (Fig. 4), mas, considerando o intervalo de confiança, apenas as áreas 1 e 5 seriam significativamente mais ricas em espécies. As curvas de rarefação ainda crescentes indicaram que um maior número amostral resultaria em um maior número de espécies amostradas para todas as áreas. 
Tabela 3 - Lista de características de espécies nativas das áreas amostradas na Reserva Biológica do Descoberto entre junho de 2019 e janeiro de 2020.

\begin{tabular}{|l|l|l|l|l|}
\hline Área & Espécies & Densidade de indivíduos regenerantes & Diversidade & Equitabilidade \\
\hline 1 & 65 & 5020 & 0.96 & 0,87 \\
\hline 2 & 44 & 66875 & 0.34 & 0,19 \\
\hline 3 & 33 & 54740 & 0.28 & 0,21 \\
\hline 4 & 36 & 65060 & 0.17 & 0,14 \\
\hline 5 & 67 & 62717 & 0.75 & 0,45 \\
\hline 6 & 20 & 83117 & 0.49 & 0,30 \\
\hline 7 & 47 & 157450 & 0.68 & 0,40 \\
\hline
\end{tabular}

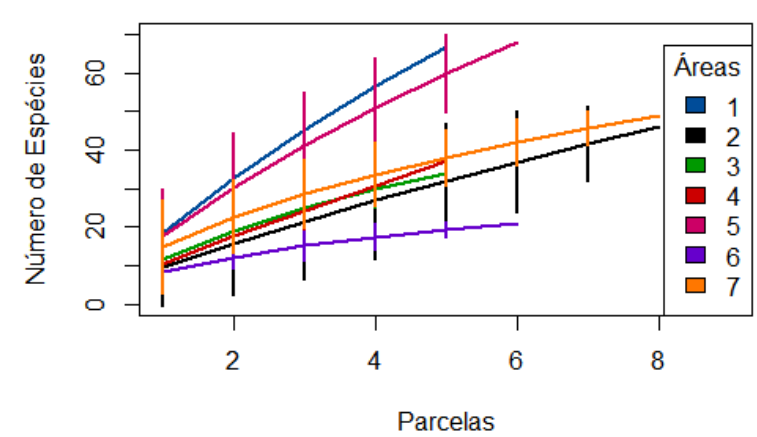

Figura 4 - Curvas de rarefação para as sete áreas da Reserva Biológica do Descoberto amostradas entre junho de 2019 e janeiro de 2020.

As áreas mais similares em termos de composição de espécies foram as áreas 1 e 2,1 e 4,1 e 6 , e 4 e 7 (SФrensen, Ss = 0,86, 0,84, 0,86 e 0,86, respectivamente). As áreas mais distintas entre si foram 3 e 4 ( $\mathrm{Ss}=0,61$ ), as demais áreas apresentaram mais alta similaridade entre elas (Ss $=0,69$ e 0,83).

\section{Discussão}

O controle de espécies invasoras é o principal obstáculo a ser superado na restauração de áreas invadidas. Espécies exóticas são capazes de alterar condições do ecossistema $e$ influenciar a trajetória da restauração, diferenciando-a da trajetória de distúrbio (Suding, 2011). Assim, mesmo o controle dos fatores de degradação, pelo controle das exóticas, pode não ser suficiente para a recuperação da área.
Pelo contrário, intervenções podem resultar em ecossistemas alternativos ou na persistência da degradação (Grootjans e Andel, 2006; Harris e Diggelen, 2006; Hobbs 1996).

Quando comparadas com a legislação do IBRAM (Instituto Brasília Ambiental), as exigências do número mínimo de espécies nativas e densidade de indivíduos nativos regenerantes foram cumpridas e superadas em todas as áreas. Por outro lado, após a primeira estação chuvosa, nenhuma das áreas alcançou todos os valores de cobertura exigidos. Apenas a área 7 atingiu o valor mínimo de cobertura total de plantas nativas. A baixa cobertura de gramíneas nativas em todas as áreas contrasta com outros estudos com semeadura direta para restaurar savanas. Gramíneas como o Andropogon fastigiatus e Schizachyrium sanguineum costumam cobrir grandes extensões de solo, sendo, muitas vezes, as espécies mais presentes nas áreas de restauração (Coutinho et al., 2019; Sampaio et al., 2015). Acredita-se que o baixo número de cobertura por essas espécies aqui obtido seja devido à época de amostragem (período de seca). Nessas condições, como foi observado em campo, as gramíneas ressecam, diminuindo sua biomassa aérea. Ainda assim, quatro das áreas apresentaram valores de cobertura de gramíneas nativas não diferentes de 30\% (mínimo exigido pela norma do IBRAM).

As árvores do Cerrado foram pouco amostradas nas áreas de estudo, possivelmente por dois motivos: a baixa quantidade de sementes plantadas em relação a outras experiências de restauração e o lento crescimento característico da maioria das espécies semeadas. Outros trabalhos que atingiram uma densidade de mais de 3000 indivíduos por hectare (mínimo exigido pela 
norma do DF) semearam uma densidade maior de espécies arbóreas, mais de 1100 sementes/ $\mathrm{m}^{2}$ (Liaffa, 2020), 1,6 vezes a mais do que foi semeado nas áreas do presente estudo. Espécies de árvores do Cerrado podem não atingir tamanhos iguais ou superiores a $30 \mathrm{~cm}$ (critério mínimo de amostragem) no período de um ano (De Oliveira et al., 2019; Pellizzaro et al., 2017; Pilon e Durigan, 2013). Em seus estudos, após a primeira estação chuvosa, Liaffa (2020) encontrou somente $17 \%$ das espécies arbóreas semeadas, todas com tamanho inferior a $10 \mathrm{~cm}$. Dessa forma, no presente trabalho, mesmo que germinados, esses indivíduos podem não ter atingido o critério mínimo ( $30 \mathrm{~cm}$ de altura) para serem incluídos nas amostragens.

Em função do baixo esforço amostral, não é possível delimitar os mecanismos exatos que podem ter levado às diferenças observadas entre as áreas. Contudo, uma hipótese levantada é que elas apresentem diferenças no resultado da restauração devido à diferente forma de semeadura, ao tempo transcorrido desde a intervenção e, principalmente, ao menor número de gradagens realizadas. A cobertura vegetal de áreas restauradas invadidas tende a variar drasticamente em poucos anos (Coutinho et al., 2019). Assim, a área 1, tendo sido semeada quase um ano antes das demais, pode ter passado por variações em sua cobertura que a diferiram das demais. Corrobora com essa hipótese a baixa densidade de Lepidaploa aurea e Stylosanthes capitata/macrocephala encontrados nessa área. Essas espécies, muito utilizadas em plantios de restauração por ocuparem rapidamente o solo (Pellizzaro et al., 2017; Sampaio et al., 2015), tendem a reduzir sua cobertura e desaparecer entre 2 a 3 anos após a semeadura (Coutinho et al,. 2019; Starr et al., 2013).

Apesar do histórico de uso das áreas não ter sido considerado um fator central neste artigo, a presença de adubação na área 5 pode ter afetado consideravelmente seus resultados. Nesse local, a cobertura por exóticas foi significativamente maior do que nas áreas 2, 3 e 4, assim como a diversidade de nativas. Os efeitos da adubação sobre as exóticas já eram esperados. No estudo de Sampaio et al., (2019), estes hipotetizaram que, em solos mais férteis, as gramíneas exóticas teriam maior crescimento, reduzindo assim a capacidade competitiva das plantas nativas.
Neste trabalho, contudo, os valores de cobertura por plantas nativas foram significativamente mais altos na área adubada do que nas demais, indicando um possível efeito da adubação nessas espécies. As espécies Stylosanthes capitata, $S$. macrocephala e Lepidaploa aurea respondem à adubação diferentemente de outras plantas nativas, para as quais a adubação não tem efeito ou reduz o crescimento (Silva et al., 2015). A área 5 , onde houve maior cobertura de Stylosanthes capitata e $S$. macrocephala, foi também a área que foi adubada. Indo além, na área adubada o grande retorno de exóticas pode ter afetado as gramíneas nativas. Damasceno et al., (2018) verificaram que gramíneas exóticas invasoras atuam com maior intensidade em espécies de igual grupo funcional, o que explicaria os baixos valores de cobertura de gramíneas nativas nessa área.

As áreas com Cerrado remanescente apresentaram alta similaridade com as demais áreas, contrário ao que seria esperado devido à esperada maior rebrota de plantas nativas. Essas áreas apresentaram um resultado da restauração muito semelhante às demais áreas de pastagem abandonada em termos de cobertura vegetal, riqueza, diversidade e densidade de regenerantes. De forma similar, a área 1, apesar de compartilhar o histórico das áreas 2, 6 e 7, apresentou resultados significativamente diferentes das demais. Esses resultados indicam a presença de outro fator atuante nas diferenças percebidas entre as áreas, o qual parece ser o preparo do solo.

Apesar das origens da regeneração natural não terem sido avaliadas neste estudo, acredita-se que a diversidade de plantas não semeadas seja proveniente de rebrota de estruturas subterrâneas. Isso especialmente para as áreas onde não havia vegetação nativa remanescente que pudesse alimentar o banco de sementes. A presença de espécies nativas não semeadas provenientes de rebrota é comum em áreas perturbadas, tendo inúmeros trabalhos verificado a importância da rebrota para a resiliência das espécies savânicas (Cava et al., 2016; Vieira et al., 2006; Durigan et al., 1998). O revolvimento do solo provoca a redução da capacidade de rebrota de espécies nativas, mas não as elimina completamente e, ao mesmo tempo, promove a germinação do banco de sementes de gramíneas invasoras. A regeneração natural é a forma mais eficiente de colonização de um ambiente degradado (Durigan et al., 1998), permitindo não só maior diversidade vegetal, 
como também a conservação do patrimônio genético da vegetação local (McKay et al., 2005). Assim, iniciativas de restauração devem sempre considerar o potencial de regeneração natural da área.

Dessa forma, em áreas ausentes de condições que favoreçam as exóticas em detrimento das nativas (ex. adubação), o preparo do solo que reduza, mas não elimine as estruturas subterrâneas das plantas, permitindo sua regeneração, pode ser uma estratégia promissora. As áreas de estudo tiveram atrasos de cronograma, o que resultou em ações de preparo do solo abaixo do recomendado em grande parte das áreas e com intervalos menores. Estudos indicam que o preparo do solo deve ser feito com antecedência de 6 meses, com 6 passagens para que se obtenha um maior sucesso na restauração por semeadura direta (Sampaio et al., 2019).

Nesse sentido, é importante que os projetos de restauração sigam um cronograma adequado à área que receberá as ações, para que não haja retorno excessivo de exóticas, o que significa uma maior necessidade de manejo após a semeadura, especialmente nos primeiros anos (Campos-Filho et al., 2013). Paralelamente, muitos estudos já indicam que o manejo das espécies exóticas invasoras deve ser de longa duração ou até mesmo permanente, sendo necessário tanto em áreas restauradas, como em áreas conservadas, devido ao constante risco de invasão (Assis et al., 2020; Coutinho et al., 2019; Suding, 201; D’Antonio \& Meyerson, 2002). A própria legislação para áreas restauradas não exige total erradicação das gramíneas exóticas, frente a dificuldade de eliminá-las, exigindo, contudo, seu controle (IBRAM, 2017).

Este trabalho confirma que a principal dificuldade na restauração é o controle das gramíneas invasoras. Para isso, há conhecimento e experiência no uso de uma série de ferramentas, que adequadamente combinadas podem resultar em um controle eficaz das invasoras, e sucesso da restauração. Entre essas ferramentas, temos o uso de herbicidas, técnica eficaz e eficiente que viabiliza a restauração em larga-escala (Campos-Filho et al., 2013), a qual pode ainda ser combinada com uso do fogo (Martins et al., 2011) e o pastejo por animais, como o gado (Buisson 2019).
A técnica a ser escolhida para que seja aplicável em larga escala deve ainda ter seu custo-benefício avaliado. Da mesma forma, deve-se monitorar as áreas em restauração por vários anos, além dos três anos comumente demandados, para melhor entender a eficácia das técnicas aplicadas.

\section{Conclusão}

A semeadura direta vem sendo progressivamente aplicada como forma de restaurar savanas neotropicais. O presente estudo revela que essa técnica pode ser capaz de atingir a exigência legal, hoje determinada por indicadores ecológicos do sucesso da restauração, se aplicada em conjunto com controle prévio ao plantio e manejo posterior das gramíneas invasoras. Os resultados aqui apresentados possuem importantes implicações não só para gestores de áreas de conservação, como para praticantes de restauração.

Nesse cenário, o conhecimento acerca do histórico da área a ser restaurada é de vital importância. A longo prazo, esse conhecimento, juntamente com ações de monitoramento, pode auxiliar a identificação de padrões e caminhos futuros, direcionando o manejo. Assim, pode-se afirmar que a semeadura direta é um dos possíveis caminhos para restaurar áreas degradadas $e$ invadidas em savanas neotropicais, atingindo as exigências legais a um baixo custo.

\section{Agradecimentos}

Ao CNPq, pela bolsa. Ao ICMBio, pelo apoio, oportunidade e disponibilização de voluntários para auxílio nas amostragens em campo. Ao Laboratório de Ecologia Vegetal da Universidade de Brasilia (EcoVeg/UNB), pela disponibilização dos materiais. À ADASA, pelo apoio logístico e disponibilização de transporte. Ao IBRAM, pelo auxilio, permissão e materiais. À Suellen, por toda sua ajuda e apoio durante o processo.

\section{Referências}

Assis GB, Pilon NAL, Siqueira MF \& Durigan G. Effectiveness and costs of invasive species control using different techniques to restore cerrado grasslands. Restoration Ecology, 2020. 
Buisson E, et al. Resilience and restoration of tropical and subtropical grasslands, savannas, and grassy woodlands. Biological Reviews, 94: 590-609, 2019.

Campos-Filho EM, Da Costa JNMN, De Sousa OL \& Junqueira RGP. Mechanized Direct-Seeding of Native Forests in Xingu, Central Brazil. Journal of Sustainable Forestry, 32: 702-727, 2013.

Cava MG de BC, Isernhagen I, Mendonça AH de \& Durigan G. Comparação de técnicas para restauração da vegetação lenhosa de Cerrado em pastagens abandonadas. Hoehnea, 43: 301-315, 2016.

Coutinho AG, Alves M, Sampaio AB, Schmidt IB \& Vieira DLM. Effects of initial functional-group composition on assembly trajectory in savanna restoration. Applied Vegetation Science, 22: 61-70, 2019.

D'Antonio C \& Meyerson LA. Exotic plant species as problems and solutions in ecological restoration: A synthesis. Restoration Ecology, 10: 703-713, 2002.

D’Antônio C \& Vitousek PM. Biological invasions by exotic grasses, the grass-fire cycle, and global change. Annual Review of Ecology and Systematics, 23: 63-87, 1992.

Damasceno G, Souza L, Pivello VR, Gorgone-Barbosa E, Giroldo PZ \& Fidelis A. Impact of invasive grasses on Cerrado under natural regeneration. Biological Invasions, 20: 3621-3629, 2018.

De Oliveira MC, Leite JB, Da Silva Galdino OP, Ogata RS, Da Silva DA \& Ribeiro JF. Survival and growth of cerrado native species after direct sowing in abandoned pasture recovery. Neotropical Biology and Conservation, 14: 313-327, 2019.

Durigan G, Contieri WA, Franco GADC \& Garrido MAO. Indução do processo de regeneração da vegetação de cerrado em área de pastagem, Assis, SP. Acta Botanica Brasilica, 12: 421-429, 1998.

Flora do Brasil 2020 em construção. Jardim Botânico do Rio de Janeiro. <http://floradobrasil.jbrj.gov.br/>. Acesso em: 21/08/2020.

Gorgone-Barbosa E, Daibes LF, Novaes RB, Pivello VR $\&$ Fidelis A. Fire cues and germination of invasive and native grasses in the Cerrado. Acta Botanica Brasilica, 34: 185-191, 2020.

Gorgone-Barbosa E, Pivello VR, Bautista S, Rissi MN \& Fidelis A. How can an invasive grass affect fire behavior in a tropical savanna? A community and individual plant level approach. Biological Invasions, 17: 423431, 2015.

Grootjans AP \& Andel JVan. 2006. Concepts in restoration ecology,p. 16-28. In: Andel JVan, Aronson J (orgs.). Restoration Ecology. Blackwell Publishing. 13p.
Harris JA \& Diggelen RVan. 2006. Ecological restoration as a project for global society, p. 3-15. In: Andel JVan, Aronson J (orgs.). Restoration Ecology. Blackwell Publishing. 13p.

Hobbs RJ. Towards a Conceptual Framework for Restoration Ecology. Restoration Ecology, 4: 93-110, 1996.

Hobbs RJ, Cramer VA. Restoration Ecology: Interventionist Approaches for Restoring and Maintaining Ecosystem Function in the Face of Rapid Environmental Change. Annual Review of Environment and Resources, 33: 39-61, 2008.

Hobbs RJ, Hallett LM, Ehrlich PR \& Mooney HA. Intervention Ecology: Applying Ecological Science in the Twenty-first Century. BioScience, 61: 442-45, 2011.

Hoffmann WA \& Haridasan M. The invasive grass, Melinis minutiflora, inhibits tree regeneration in a Neotropical savanna. Austral Ecology, 33: 29-36, 2008.

Hothorn T, Bretz F \& Westfall P. Simultaneous inference in general parametric models. Biometrical Journal, 50(3): 346-63, 2008.

IBRAM (Instituto Brasília Ambiental). 2018. Indicadores Ecológicos Para a Recomposição da Vegetação Nativa no Distrito Federal. Nota Técnica. 23p.

Kettenring KM \& Adams CR. Lessons learned from invasive plant control experiments: A systematic review and meta-analysis. Journal of Applied Ecology, 48: 970-979, 2011.

Liaffa ABS. 2020. Restauração ecológica no Cerrado: intenso preparo de solo e alta densidade de semeadura não eliminam a necessidade de erradicação de gramíneas exóticas invasoras. Dissertação (Mestrado em Ecologia). Universidade de Brasília. 67p.

Martins CR, Hay JDV, Walter BMT, Proença CEB \& Vivaldi LJ. Impacto da invasão e do manejo do capim-gordura (Melinis minutiflora) sobre a riqueza $e$ biomassa da flora nativa do Cerrado sentido restrito. Brazilian Journal of Botany, 34(1): 73-90, 2011.

McKay JK, Christian CE, Harrison S \& Rice KJ. "How local is local?" - A review of practical and conceptual issues in the genetics of restoration. Restoration Ecology, 13: 432-440, 2005.

Oksanen J et al. 2019. vegan: Community Ecology Package. R package version 2.5-6. <https:/CRAN.Rproject.org/package = vegan $>$. Acesso em: 20/10/19.

Palma AC \& Laurance SGW. A review of the use of direct seeding and seedling plantings in restoration: What do we know and where should we go? Applied Vegetation Science, 18: 561-568, 2015. 
Pellizzaro KF et al. "Cerrado" restoration by direct seeding: field establishment and initial growth of 75 trees, shrubs and grass species. Revista Brasileira de Botanica, 40: 681-693 2017.

Pilon N \& Durigan G. Critérios para indicação de espécies prioritárias para a restauração da vegetação de cerrado. Scientia Forestalis, 41: 389-399, 2013.

R Core Team (2018). R: A language and environment for statistical computing. R Foundation for

Statistical Computing, Vienna, Austria. < https:// www.R-project.org > Acesso em: 20/10/19.

Reatto A, Martins E de S, Farias MFR, Silva AV da \& Carvalho Junior AO de. Mapa pedológico digital: SIG atualizado do Distrito Federal escala 1:100.000 e uma síntese do texto explicativo 2004.

Ruiz-Jaen MC \& Aide TM. Restoration success: How is it being measured? Restoration Ecology, 13: 569-577, 2005.

Sampaio AB et al. 2015. Guia de restauração do Cerrado. 1 ed. Embrapa Cerrado. 33p.

Sampaio AB \& Schmidt IB. Espécies exóticas invasoras em unidades de conservação federais do Brasil. Biodiversidade Brasileira 32-49, 2014.

Sampaio $\mathrm{AB}$ et al. Lessons on direct seeding to restore Neotropical savanna. Ecological Engineering, 138: 148-154, 2019.

Schmidt IB et al. Community-based native seed production for restoration in Brazil - the role of science and policy. Plant Biology, 21: 389-397, 2018.

Schmidt IB, Ferreira MC, Sampaio AB, Walter BMT, Vieira DLM \& Holl KD. Tailoring restoration interventions to the grassland-savanna-forest complex in central Brazil. Restoration Ecology, 27: 942-948, 2019.

Sherman GE et al. 2011. Quantum GIS User Guide - Version 1.7 "Wroclaw". <http://download.osgeo. org/qgis/doc/manual/qgis-1.7.0_user_guide_en.pdf $>$. Acesso em: 20/10/19.

Silva RRP, Oliveira DR, da Rocha GPE \& Vieira DLM. Direct seeding of Brazilian savanna trees: Effects of plant cover and fertilization on seedling establishment and growth. Restoration Ecology 23: 393-401, 2015.

Silveira FAO, Arruda AJ, Bond W, Durigan G, Fidelis A, Kirkman K, Oliveira RS, Overbeck GE, Sansevero JBB, Siebert F, Siebert SJ, Young TP \& Buisson E. Mythbusting tropical grassy biome restoration. Restoration Ecology, 28: 1067-1073, 2020.

Sousa A de P \& Vieira DLM. 2017. Protocolo de Monitoramento da Recomposição da Vegetação Nativa no Distrito Federal. WWF. 8p.
Starr CR, Corrêa RS, Filgueiras T de S, Hay JDV \& dos Santos PF. Plant colonization in a gravel mine revegetated with Stylosanthes spp. in a Neotropical savanna. Landscape and Ecological Engineering, 9: 189-201, 2013.

Suding KN. Toward an Era of Restoration in Ecology: Successes, Failures, and Opportunities Ahead. Annual Review of Ecology, Evolution, and Systematics, 42: 465-487, 2011.

Uramoto K, Walder JMM \& Zucchi RA. Análise quantitativa e distribuição de populações de espécies de anastrepha (Diptera: Tephritidae) no Campus Luiz de Queiroz, Piracicaba, SP. Neotropical Entomology, 34: 33-39, 2005.

Vieira DLM, Scariot A, Sampaio AB \& Holl KD. Tropical dry-forest regeneration from root suckers in Central Brazil. Journal of Tropical Ecology, 22: 353-357, 2006.

Wortley L, Hero JM \& Howes M. Evaluating ecological restoration success: A review of the literature. Restoration Ecology, 21: 537-543, 2013.

\section{Material Suplementar}

Espécies semeadas e identificadas em áreas restauradas na Reserva Biológica do Descoberto, entre junho de 2019 e janeiro de 2020. EA (Espécies amostradas) indica as espécies amostradas na área: S (sim) e N (não) indicam a presença ou ausência das espécies nas parcelas. Gramíneas semeadas foram indicadas por (-) pois, apesar de terem sido amostradas gramíneas nas parcelas, não foi possível identificá-las não havendo, portanto, certeza quanta à presença de cada espécie. 


\begin{tabular}{|c|c|c|c|c|c|c|}
\hline \multirow{2}{*}{ Nome científico } & \multirow{2}{*}{ Família } & \multirow{2}{*}{$\begin{array}{l}\text { Forma de } \\
\text { vida }\end{array}$} & \multicolumn{3}{|c|}{ Plantio (sementes/m²) } & \multirow{2}{*}{ EA } \\
\hline & & & Area 1 & $\begin{array}{c}\text { Area } 2 \\
\text { a } 7\end{array}$ & $\begin{array}{c}\text { Area } 1 \\
\text { adensamento }\end{array}$ & \\
\hline Pfaffia tuberosa (Spreng.) Hicken & Amaranthaceae & Subarbusto & - & - & - & S \\
\hline Anacardium humile A.St.-Hil. & Anacardiaceae & Arbusto & - & - & 0,43 & $\mathrm{~s}$ \\
\hline Myracrodruon urundeuva M. Allemão & Anacardiaceae & Árvore & - & 10,24 & 36,08 & $\mathrm{~S}$ \\
\hline Anacardium occidentale $\mathrm{L}$. & Anacardiaceae & Árvore & - & 0,21 & - & $\mathrm{N}$ \\
\hline Astronium fraxinifolium Schott & Anacardiaceae & Árvore & - & 3,20 & - & $\mathrm{N}$ \\
\hline Mandevilla longiflora (Desf.) Pichon & Apocynaceae & Erva & - & - & - & $\mathrm{s}$ \\
\hline Arecaceae sp. & Arecaceae & Árvore & - & - & - & S \\
\hline Achyrocline satureioides (Lam.) DC. & Asteraceae & Arbusto & - & - & - & $\mathrm{S}$ \\
\hline $\begin{array}{l}\text { Ageratum fastigiatum (Gardner) R.M.King \& } \\
\text { H.Rob. }\end{array}$ & Asteraceae & Subarbusto & - & - & - & $\mathrm{S}$ \\
\hline Asteraceae sp. 1 & Asteraceae & Subarbusto & - & - & - & S \\
\hline Asteraceae sp. 2 & Asteraceae & Subarbusto & - & - & - & $\mathrm{s}$ \\
\hline Asteraceae sp. 3 & Asteraceae & Subarbusto & - & - & - & S \\
\hline Asteraceae sp. 4 & Asteraceae & Subarbusto & - & - & - & $\mathrm{S}$ \\
\hline Asteraceae sp. 5 & Asteraceae & Subarbusto & - & - & - & $\mathrm{s}$ \\
\hline Asteraceae sp. 6 & Asteraceae & Subarbusto & - & - & - & $\mathrm{s}$ \\
\hline Ayapana amygdalina (Lam.) R.M.King \& H.Rob. & Asteraceae & Subarbusto & - & - & - & S \\
\hline Baccharis dracunculifolia DC. & Asteraceae & Arbusto & - & - & - & $\mathrm{S}$ \\
\hline Bidens pilosa $\mathrm{L}$. & Asteraceae & Erva & - & - & - & $\mathrm{S}$ \\
\hline Chresta sphaerocephala DC. & Asteraceae & Arbusto & - & - & - & $\mathrm{S}$ \\
\hline Emilia fosbergii Nicolson & Asteraceae & Erva & - & - & - & $\mathrm{S}$ \\
\hline Lepidaploa aurea (Mart. ex DC.) H.Rob. & Asteraceae & Arbusto & - & 244,77 & 274,81 & $\mathrm{~S}$ \\
\hline Lessingianthus bardanoides (Less.) H.Rob. & Asteraceae & Subarbusto & - & - & - & $\mathrm{s}$ \\
\hline Porophyllum lanceolatum DC. & Asteraceae & Erva & - & - & - & $\mathrm{S}$ \\
\hline Pterocaulon rugosum (Vahl) Malme & Asteraceae & Erva & - & - & - & $S$ \\
\hline Sonchus oleraceus L. & Asteraceae & Erva & - & - & - & $\mathrm{s}$ \\
\hline Tridax procumbens $\mathrm{L}$. & Asteraceae & Erva & - & - & - & $\mathrm{s}$ \\
\hline Vernonanthura brasiliana (L.) H.Rob. & Asteraceae & Arbusto & - & - & - & $\mathrm{S}$ \\
\hline $\begin{array}{l}\text { Vernonanthura polyanthes (Sprengel) Vega \& } \\
\text { Dematteis }\end{array}$ & Asteraceae & Arbusto & 5,24 & 76,67 & 0,87 & S \\
\hline Eremanthus glomerulatus Less. & Asteraceae & Árvore & - & 3,25 & - & $\mathrm{N}$ \\
\hline $\begin{array}{l}\text { Anemopaegma arvense (Vell.) Stellfeld ex de } \\
\text { Souza }\end{array}$ & Bignoniaceae & Subarbusto & - & - & - & S \\
\hline Heliotropium indicum L. & Bignoniaceae & Erva & - & - & - & $\mathrm{S}$ \\
\hline Jacaranda ulei Bureau \& K.Schum. & Bignoniaceae & Arbusto & - & - & - & $\mathrm{s}$ \\
\hline $\begin{array}{l}\text { Tabebuia aurea (Silva Manso) Benth. \& Hook.f. } \\
\text { ex S.Moore }\end{array}$ & Bignoniaceae & Árvore & - & 0,28 & - & $\mathrm{N}$ \\
\hline Cochlospermum regium (Mart. ex Schrank) Pilg. & Bixaceae & Arbusto & - & - & - & S \\
\hline Bromeliaceae sp. 1 & Bromeliaceae & Erva & - & - & - & S \\
\hline Kielmeyera coriacea Mart. \& Zucc. & Calophyllaceae & Árvore & - & 1,71 & - & $\mathrm{N}$ \\
\hline Caryocar brasiliense Cambess. & Caryocaraceae & Árvore & - & 0,07 & - & $\mathrm{N}$ \\
\hline
\end{tabular}




\begin{tabular}{|c|c|c|c|c|c|c|}
\hline Buchenavia tomentosa Eichler & Combretaceae & Árvore & - & 0,38 & - & $\mathrm{N}$ \\
\hline Terminalia argentea Mart. & Combretaceae & Árvore & - & 1,06 & - & $\mathrm{N}$ \\
\hline Terminalia fagifolia Mart. & Combretaceae & Árvore & - & 0,88 & - & $\mathrm{N}$ \\
\hline Cyperaceae sp. 1 & Cyperaceae & Erva & - & - & - & $\mathrm{S}$ \\
\hline Cyperaceae sp. 2 & Cyperaceae & Erva & - & - & - & $\mathrm{S}$ \\
\hline Croton didrichsenii G.L.Webster & Euphorbiaceae & Subarbusto & - & - & - & $\mathrm{S}$ \\
\hline Arachis pintoi Krapov. \& W.C.Greg. & Fabaceae & Erva & - & - & - & $\mathrm{S}$ \\
\hline Bauhinia sp. 1 & Fabaceae & Árvore & - & - & - & $\mathrm{S}$ \\
\hline Bauhinia sp. 2 & Fabaceae & Árvore & - & - & - & $\mathrm{S}$ \\
\hline Calliandra dysantha Benth. & Fabaceae & Subarbusto & - & - & - & $\mathrm{S}$ \\
\hline Calopogonium mucunoides Desv. & Fabaceae & Liana & - & - & - & $\mathrm{S}$ \\
\hline Fabacea sp. 1 & Fabaceae & Arbusto & - & & - & $\mathrm{S}$ \\
\hline Fabacea sp. 2 & Fabaceae & Arbusto & - & & - & $\mathrm{S}$ \\
\hline Fabacea sp. 3 & Fabaceae & Arbusto & - & & - & $\mathrm{S}$ \\
\hline Fabacea sp. 4 & Fabaceae & Erva & - & & - & $\mathrm{S}$ \\
\hline Fabacea sp. 5 & Fabaceae & Arbusto & - & & - & $\mathrm{S}$ \\
\hline Mimosa claussenii Benth. & Fabaceae & Arbusto & - & - & - & $\mathrm{S}$ \\
\hline Mimosa decorticans Barneby & Fabaceae & Arbusto & - & - & - & $\mathrm{S}$ \\
\hline Mimosoideae sp. 1 & Fabaceae & Arbusto & - & & - & $\mathrm{S}$ \\
\hline Mimosoideae sp. 2 & Fabaceae & Arbusto & - & & - & $\mathrm{S}$ \\
\hline Mimosoideae sp. 3 & Fabaceae & Arbusto & - & & - & $\mathrm{S}$ \\
\hline Mimosoideae sp. 4 & Fabaceae & Arbusto & - & & - & $\mathrm{S}$ \\
\hline Mimosoideae sp. 5 & Fabaceae & Arbusto & - & & - & $\mathrm{S}$ \\
\hline Mimosoideae sp. 6 & Fabaceae & Arbusto & - & & - & S \\
\hline Mimosoideae sp. 7 & Fabaceae & Arbusto & - & & - & $\mathrm{S}$ \\
\hline Mimosoideae sp. 8 & Fabaceae & Arbusto & - & & - & $\mathrm{s}$ \\
\hline Mimosoideae sp. 9 & Fabaceae & Arbusto & - & & - & $\mathrm{S}$ \\
\hline Senna alata (L.) Roxb. & Fabaceae & Arbusto & - & 3,55 & 4,08 & $\mathrm{~S}$ \\
\hline $\begin{array}{l}\text { * Stylosanthes capitata Vogel / Stylosanthes ma- } \\
\text { crocephala M.B.Ferreira \& Sousa Costa }\end{array}$ & Fabaceae & Subarbusto & - & 185,19 & - & $S$ \\
\hline Stylosanthes sp. 1 & Fabaceae & Subarbusto & - & - & - & $\mathrm{S}$ \\
\hline Stylosanthes sp. 2 & Fabaceae & Subarbusto & - & - & - & $\mathrm{s}$ \\
\hline Anadenanthera colubrina (Vell.) Brenan & Fabaceae & Árvore & - & 0,39 & - & $\mathrm{N}$ \\
\hline Andira vermifuga (Mart.) Benth. & Fabaceae & Árvore & - & 0,02 & - & $\mathrm{N}$ \\
\hline Dimorphandra mollis Benth. & Fabaceae & Árvore & - & 0,62 & - & $\mathrm{N}$ \\
\hline Dipteryx alata Vogel & Fabaceae & Árvore & - & 0,90 & 0,54 & $\mathrm{~N}$ \\
\hline Enterolobium contortisiliquum (Vell.) Morong & Fabaceae & Árvore & - & 0,97 & - & $\mathrm{N}$ \\
\hline Hymenaea courbaril L. & Fabaceae & Árvore & - & 0,15 & - & $\mathrm{S}$ \\
\hline Hymenaea stigonocarpa Mart. ex Hayne & Fabaceae & Árvore & - & 0,07 & - & $\mathrm{N}$ \\
\hline Machaerium opacum Vogel & Fabaceae & Árvore & - & 0,06 & - & $\mathrm{N}$ \\
\hline Hyptis cardiophylla Pohl ex Benth. & Lamiaceae & Subarbusto & - & - & - & S \\
\hline $\begin{array}{l}\text { Peltaea polymorpha (A.St.-Hil.) Krapov. \& } \\
\text { Cristóbal }\end{array}$ & Malvaceae & Erva & - & - & - & S \\
\hline Sida rhombifolia L. & Malvaceae & Erva & - & - & - & S \\
\hline Waltheria indica $\mathrm{L}$. & Malvaceae & Subarbusto & - & - & - & $\mathrm{S}$ \\
\hline
\end{tabular}




\begin{tabular}{|c|c|c|c|c|c|c|}
\hline Guazuma ulmifolia Lam. & Malvaceae & Árvore & - & 36,13 & - & $\mathrm{N}$ \\
\hline Desmoscelis villosa (Aubl.) Naudin & $\begin{array}{l}\text { Melastomata- } \\
\text { ceae }\end{array}$ & Erva & - & - & - & $\mathrm{S}$ \\
\hline Brosimum gaudichaudii Trécul & Moraceae & Arbusto & - & 0,02 & - & $\mathrm{N}$ \\
\hline Ouratea floribunda (A.St.-Hil.) Engl. & Ochnaceae & Subarbusto & - & - & - & $\mathrm{S}$ \\
\hline Oxalis densifolia Mart. \& Zucc. ex Zucc. & Oxalidaceae & Subarbusto & - & - & - & $\mathrm{S}$ \\
\hline Poaceae sp. 1 & Poaceae & Erva & - & & - & $\mathrm{S}$ \\
\hline Poaceae sp. 2 & Poaceae & Erva & - & & - & $\mathrm{S}$ \\
\hline Poaceae sp. 3 & Poaceae & Erva & - & & - & $\mathrm{S}$ \\
\hline Poaceae sp. 4 & Poaceae & Erva & - & & - & $\mathrm{S}$ \\
\hline Poaceae sp. 5 & Poaceae & Erva & - & & - & $\mathrm{S}$ \\
\hline Poaceae sp. 6 & Poaceae & Erva & - & & - & $\mathrm{S}$ \\
\hline Poaceae sp. 7 & Poaceae & Erva & - & & - & $\mathrm{S}$ \\
\hline Poaceae sp. 8 & Poaceae & Erva & - & & - & $\mathrm{S}$ \\
\hline Poaceae sp. 9 & Poaceae & Erva & - & & - & $\mathrm{S}$ \\
\hline Poaceae sp. 10 & Poaceae & Erva & - & & - & $\mathrm{S}$ \\
\hline Andropogon fastigiatus Sw. & Poaceae & Erva & 151,67 & 94,14 & 151,67 & - \\
\hline Aristida flaccida Trin. \& Rupr. & Poaceae & Erva & - & 15,00 & 19,96 & - \\
\hline Aristida gibbosa (Nees) Kunth & Poaceae & Erva & - & 22,52 & 28,70 & - \\
\hline Aristida riparia Trin. & Poaceae & Erva & 186,92 & 27,31 & 46,73 & - \\
\hline Axonopus aureus P. Beauv. & Poaceae & Erva & 39,92 & - & 59,88 & - \\
\hline Axonopus barbigerus (Kunth) Hitchc. & Poaceae & Erva & 39,92 & 38,33 & 59,88 & - \\
\hline $\begin{array}{l}\text { Hyparrhenia bracteata (Humb. \& Bonpl. Ex } \\
\text { Wild) Stapf }\end{array}$ & Poaceae & Erva & - & 16,69 & 12,49 & - \\
\hline Loudetiopsis chrysothrix (Nees) Conert & Poaceae & Erva & 4,36 & 7,28 & 6,54 & - \\
\hline Paspalum stellatum Humb. \& Bonpl. ex Flüggé & Poaceae & Erva & - & - & 35,21 & - \\
\hline Schizachyrium sanguineum (Retz.) Alston & Poaceae & Erva & - & 44,43 & 29,56 & - \\
\hline Trachypogon spicatus (L.f.) Kuntze & Poaceae & Erva & 1,36 & - & 2,04 & - \\
\hline Borreria sp. 1 & Rubiaceae & Erva & - & - & - & $\mathrm{S}$ \\
\hline Borreria sp. 2 & Rubiaceae & Erva & - & - & - & $\mathrm{S}$ \\
\hline Borreria spinosa Cham. et Schltdl. & Rubiaceae & Erva & - & - & - & $\mathrm{S}$ \\
\hline Sabicea brasiliensis Wernham & Rubiaceae & Subarbusto & - & - & - & $\mathrm{S}$ \\
\hline Casearia sylvestris Sw. & Salicaceae & Arbusto & - & - & - & $\mathrm{S}$ \\
\hline Magonia pubescens A.St.-Hil. & Sapindaceae & Árvore & - & 0,12 & - & $\mathrm{N}$ \\
\hline Simarouba versicolor A.St.-Hil. & Simaroubaceae & Árvore & - & 0,03 & - & $\mathrm{N}$ \\
\hline Smilax goyazana A.DC. & Smilacaceae & Arbusto & - & - & - & $\mathrm{S}$ \\
\hline Physalis angulata $\mathrm{L}$. & Solanaceae & Erva & - & - & - & $\mathrm{S}$ \\
\hline Solanum lycocarpum A.St.-Hil. & Solanaceae & Arbusto & - & 1,39 & 2,70 & $\mathrm{~S}$ \\
\hline Lantana camara L. & Verbenaceae & Arbusto & - & - & - & $\mathrm{S}$ \\
\hline
\end{tabular}

* As duas espécies de Stylosanthes foram semeadas como mistura de sementes em torno 50\% de cada espécie, comprada da marca Boi Gordo. Os números no nome das espécies indicam diferentes espécies não identificadas de um mesmo gênero ou de uma determinada família. 


\section{Biodiversidade Brasileira - BioBrasil.}

Edição Temática: PIBIC

n. 1,2022

http://www.icmbio.gov.br/revistaeletronica/index.php/BioBR

Biodiversidade Brasileira é uma publicação eletrônica científica do Instituto Chico Mendes de

Conservação da Biodiversidade (ICMBio) que tem como objetivo fomentar a discussão e a disseminação de experiências em conservação e manejo, com foco em unidades de conservação $e$ espécies ameaçadas.

ISSN: 2236-2886 